\title{
Approximate Solutions of Singular Two-Point BVPs Using Legendre Operational Matrix of Differentiation
}

\author{
A. Sami Bataineh, ${ }^{1}$ A. K. Alomari, ${ }^{2}$ and I. Hashim ${ }^{3}$ \\ ${ }^{1}$ Department of Mathematics, Faculty of Science, Al-Balqa' Applied University, Al Salt 19117, Jordan \\ ${ }^{2}$ Department of Mathematics, Faculty of Science, Hashemite University, Zarqa 13115, Jordan \\ ${ }^{3}$ School of Mathematical Sciences, Universiti Kebangsaan Malaysia, 43600 Bangi, Selangor, Malaysia \\ Correspondence should be addressed to I. Hashim; ishak_h@ukm.my
}

Received 22 January 2013; Accepted 17 March 2013

Academic Editor: Sazzad Chowdhury

Copyright (C) 2013 A. Sami Bataineh et al. This is an open access article distributed under the Creative Commons Attribution License, which permits unrestricted use, distribution, and reproduction in any medium, provided the original work is properly cited.

Exact and approximate analytical solutions of linear and nonlinear singular two-point boundary value problems (BVPs) are obtained for the first time by the Legendre operational matrix of differentiation. Different from other numerical techniques, shifted Legendre polynomials and their properties are employed for deriving a general procedure for forming this matrix. The accuracy of the technique is demonstrated through several linear and nonlinear test examples.

\section{Introduction}

In this work, we consider the singular two-point boundary value problems (BVPs) of the type

$$
\begin{aligned}
\frac{1}{p(x)} u^{\prime \prime}(x)+\frac{1}{q(x)} u^{\prime}(x)+\frac{1}{r(x)}(u(x))^{n} & =g(x), \\
0 & <x \leq 1,
\end{aligned}
$$

subject to the boundary conditions

$$
u(0)=\alpha_{1}, \quad u(1)=\beta,
$$

or

$$
u^{\prime}(0)=\alpha_{2}, \quad u(1)=\beta,
$$

where $p, q, r$, and $g$ are continuous functions on $(0,1]$, and the parameters $\alpha_{1}, \alpha_{2}, \beta$ are real constants.

Problems of form (1) and (2) have been studied in many areas of science and engineering, for example, fluid mechanics, quantum mechanics, optimal control, chemical reactor theory, aerodynamics, reaction-diffusion process, geophysics, and so forth. Exact/approximate solutions of these problems are of great importance due to their wide applications in scientific research. Singular BVPs have been studied by several authors. Bataineh et al. [1] used the modified homotopy analysis method (MHAM) to search for approximate solutions of a certain class of singular two-point BVPs. Ravi Kanth and Aruna. [2] and Lu [3] used differential transform method (DTM) and variational iteration method (VIM), respectively, for solving singular two-point boundary value problems. Abu-Zaid and Gebeily [4] provided a finite difference approximation to the solution of the above problems. Ravi Kanth and Reddy [5] presented a method based on cubic splines for solving a class of singular two-point BVPs. The existence of a unique solution of (1) and (2) was discussed in [4].

Legendre operational matrix of differentiation, first proposed by Saadatmandi and Dehghan [6], is a powerful method for solving linear and nonlinear problems. They extended the application of Legendre polynomials to solve fractional differential equations. Recently, Pandey et al. [7] employed the Legendre operational matrix of differentiation to solve Lane-Emden type equations. Most recently, Kazem et al. [8] constructed a general formulation for the fractionalorder Legendre functions to obtain the solution of fractionalorder differential equations. To the best of the authors' knowledge, the present work demonstrates for the first time 
the applicability of the method of Legendre operational matrix of differentiation for obtaining the exact/approximate solutions of the singular two-point BVPs of the type (1) and (2). Several examples are studied to demonstrate the capability of the method.

\section{Legendre Polynomials and Operational Matrix of Differentiation}

The $m$ th-order Legendre polynomials, $L_{m}(z)$, on the interval $[-1,1]$ are defined as

$$
\begin{gathered}
L_{0}(z)=1, \\
L_{1}(z)=z, \\
L_{m+1}(z)=\frac{2 m+1}{m+1} z L_{m}(z)-\frac{m}{m+1} z L_{m-1}(z), \\
m=1,2, \ldots .
\end{gathered}
$$

These polynomials on the interval $z \in[0,1]$, so-called shifted Legendre polynomials, can be defined by introducing the change of variable $z=2 x-1$. The shifted Legendre polynomials $L_{m}(2 x-1)$ denoted by $P_{m}(x)$ can be obtained as

$$
\begin{array}{r}
P_{m+1}(x)=\frac{(2 m+1)(2 x-1)}{(m+1)} P_{m}(x)-\frac{m}{m+1} P_{m-1}(x), \\
m=1,2, \ldots,
\end{array}
$$

where $P_{0}(x)=1$ and $P_{1}(x)=2 x-1$. The analytic form of the shifted Legendre polynomial $P_{m}(x)$ of degree $m$ is given by

$$
P_{m}(x)=\sum_{i=1}^{m}(-1)^{m+i} \frac{(m+i) ! x^{i}}{(m-i)(i !)^{2}} .
$$

Note that $P_{m}(0)=(-1)^{m}$ and $P_{m}(1)=1$ satisfy the orthogonality condition

$$
\int_{0}^{1} P_{m}(x) P_{j}(x) d x= \begin{cases}\frac{1}{2 m+1} & \text { for } m=j \\ 0 & \text { for } m \neq j\end{cases}
$$

A function $u(x)$ square integrable in $[0,1]$ may be expressed in terms of shifted Legendre polynomials as

$$
u(x)=\sum_{j=0}^{\infty} c_{j} P_{j}(x)
$$

where the coefficients $c_{j}$ are given by

$$
c_{j}=(2 j+1) \int_{0}^{1} u(x) P_{j}(x) d x, \quad j=1,2 \ldots
$$

In practice, we consider the $(m+1)$-term-shifted Legendre polynomial so that

$$
u(x)=\sum_{j=0}^{m} c_{j} P_{j}(x)=C^{T} \phi(x),
$$

where the shifted Legendre coefficient vector $C$ and the shifted Legendre vector $\phi(x)$ are given by

$$
\begin{gathered}
C^{T}=\left[c_{0}, c_{1}, \ldots, c_{m}\right], \\
\phi(x)=\left[P_{0}(x), P_{1}(x), \ldots, P_{m}(x)\right]^{T} .
\end{gathered}
$$

The derivative of the vector $\phi(x)$ can be expressed as

$$
\begin{gathered}
\frac{d \phi(x)}{d x}=D^{1} \phi(x), \quad \frac{d^{2} \phi(x)}{d x^{2}}=\left(D^{1}\right)^{2} \phi(x), \ldots, \\
\frac{d^{n} \phi(x)}{d x^{n}}=\left(D^{1}\right)^{n} \phi(x),
\end{gathered}
$$

where $D^{1}$ is the $(m+1) \times(m+1)$ operational matrix of derivative. A general method of constructing such operational matrix of derivative could be presented as follows.

(1) Differentiate analytically some polynomials of first degree,

(2) express these derivatives as a linear combination of polynomials of lower degree, and

(3) find a general formula.

Now, the general formula of the operational matrix of derivative $D^{1}$ is given by

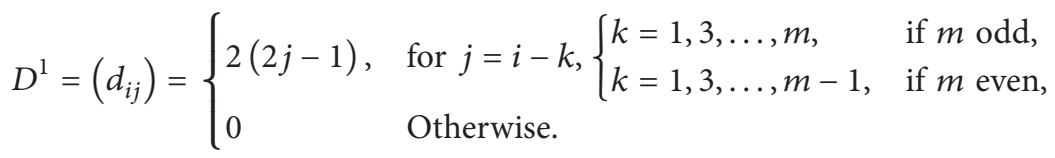

For example, for odd $m$ we have

$$
\left(\begin{array}{cccccccc}
0 & 0 & 0 & 0 & \cdots & 0 & 0 & 0 \\
2 & 0 & 0 & 0 & \cdots & 0 & 0 & 0 \\
0 & 6 & 0 & 0 & \cdots & 0 & 0 & 0 \\
\vdots & \vdots & \vdots & \vdots & \vdots & \vdots & \vdots & \vdots \\
2 & 0 & 10 & 0 & \cdots & (2 m-3) & 0 & 0 \\
0 & 6 & 0 & 14 & \cdots & 0 & (2 m-1) & 0
\end{array}\right)
$$

\section{Applications of the Operational Matrix of Derivative}

To solve (1) and (2) by means of the operational matrix of derivative method [6], we approximate $(u(x))^{n}$ and $g(x)$ by the shifted Legendre polynomials as

$$
\begin{gathered}
(u(x))^{n} \simeq\left(C^{T} \phi(x)\right)^{n}, \\
g(x) \simeq G^{T} \phi(x),
\end{gathered}
$$


where the vector $G^{T}=\left[g_{0}(x), \ldots, g_{m}(x)\right]^{T}$ represents the nonhomogenous term. By using (12), (15), and (16) we have

$$
\begin{gathered}
u^{\prime \prime}(x) \simeq C^{T}\left(D^{1}\right)^{2} \phi(x), \\
u^{\prime}(x) \simeq C^{T} D^{1} \phi(x) .
\end{gathered}
$$

Employing (15)-(18), the residual $\mathfrak{R}(x)$ for (1) can be written as

$$
\begin{aligned}
\mathfrak{R}(x) \simeq & \frac{1}{p(x)} C^{T}\left(D^{1}\right)^{2} \phi(x)+\frac{1}{q(x)} C^{T} D^{1} \phi(x) \\
& +\frac{1}{r(x)}\left(C^{T} \phi(x)\right)^{n}-G^{T} \phi(x) .
\end{aligned}
$$

Now, finding the solution $u(x)$ given in (10) can be divided into two cases: linear and nonlinear.

3.1. Linear Case. For $n=1$, we generate $m-1$ linear equations as in a typical tau method [9] by applying

$$
\int_{0}^{1} \Re(x) P_{j}(x) d x=0, \quad j=0,1, \ldots, m-2 .
$$

Also, by substituting boundary conditions (2) and (3) into (15) and (18) we have

$$
u(0)=C^{T} \phi(0)=\alpha_{1}, \quad u(1)=C^{T} \phi(1)=\beta,
$$

or

$$
u^{\prime}(0)=C^{T} D^{1} \phi(0)=\alpha_{2}, \quad u(1)=C^{T} \phi(1)=\beta,
$$

Equations (20)-(22) generate $(m+1)$ set of linear equations, respectively. These linear equations can be solved for unknown coefficients of the vector $C$. Consequently, $u(x)$ given in (15) can be easily calculated.

3.2. Nonlinear Case. For $n=2,3, \ldots$, we first collocate $(19)$ at $(m-1)$ points. For suitable collocation points, we use the first $(m-1)$ shifted Legendre roots of $P_{m+1}(x)$. These equations together with (21) or (22) generate $(m+$ 1 ) nonlinear equations which can be solved using Newton's iterative method. Consequently, $u(x)$ given in (10) can be calculated.

To illustrate the effectiveness of the presented method, we will consider the following examples of singular two-point BVPs.

Example 1. We first consider the linear singular two-point BVP $[1,10]$,

$$
u^{\prime \prime}(x)+\frac{1}{x} u^{\prime}(x)+u(x)=g(x), \quad 0<x \leq 1,
$$

subject to the boundary conditions of the form (2)

$$
u(0)=0, \quad u(1)=0 .
$$

The exact solution of (23) subject to (24) in the case of $g(x)=$ $4-9 x+x^{2}-x^{3}$ is

$$
u(x)=x^{2}-x^{3}
$$

To solve (23) and (24) we apply the technique described in Section 3.1. With $m=3$, we approximate the solution as

$$
\begin{aligned}
u(x)= & c_{0} P_{0}(x)+c_{1} P_{1}(x) \\
& +c_{2} P_{2}(x)+c_{3} P_{3}(x) \\
= & C^{T} \phi(x) .
\end{aligned}
$$

According to (14), we have

$$
D^{1}=\left(\begin{array}{cccc}
0 & 0 & 0 & 0 \\
2 & 0 & 0 & 0 \\
0 & 6 & 0 & 0 \\
2 & 0 & 10 & 0
\end{array}\right), \quad\left(D^{1}\right)^{2}=\left(\begin{array}{cccc}
0 & 0 & 0 & 0 \\
0 & 0 & 0 & 0 \\
12 & 0 & 0 & 0 \\
0 & 60 & 0 & 0
\end{array}\right) .
$$

Therefore, using (20) we obtain

$$
\begin{aligned}
& \frac{19}{20}+\frac{1}{2} c_{0}+\frac{13}{6} c_{1}+6 c_{2}+12 c_{3}=0, \\
& \frac{49}{60}+\frac{1}{6} c_{0}+\frac{1}{6} c_{1}+\frac{61}{15} c_{2}+10 c_{3}=0 .
\end{aligned}
$$

Now, from (21) we have

$$
\begin{aligned}
& c_{0}-c_{1}+c_{2}-c_{3}=0, \\
& c_{0}+c_{1}+c_{2}+c_{3}=0 .
\end{aligned}
$$

Solving the linear system (28)-(29) yields

$$
c_{0}=\frac{1}{12}, \quad c_{1}=\frac{1}{20}, \quad c_{3}=-\frac{1}{12}, \quad c_{4}=-\frac{1}{20} .
$$

Thus,

$$
\begin{aligned}
u(x) & =\left(\begin{array}{llll}
\frac{1}{12} & \frac{1}{20} & -\frac{1}{12} & -\frac{1}{20}
\end{array}\right)\left(\begin{array}{c}
1 \\
2 x-1 \\
6 x^{2}-6 x+1 \\
20 x^{3}-30 x^{2}+12 x-1
\end{array}\right) \\
& =x^{2}-x^{3},
\end{aligned}
$$

which is the exact solution (25).

Example 2. Consider the linear singular two-point BVPs [1],

$$
\begin{aligned}
\left(1-\frac{x}{2}\right) u^{\prime \prime}(x)+\frac{3}{2}\left(\frac{1}{x}-1\right) u^{\prime}(x)+\left(\frac{x}{2}-1\right) u(x) & =g(x), \\
0 & <x \leq 1,
\end{aligned}
$$

subject to the boundary conditions of the form (3)

$$
u^{\prime}(0)=0, \quad u(1)=0 .
$$


The exact solution of (32) subject to (33) in the case

$$
g(x)=5-\frac{29 x}{2}+\frac{13 x^{2}}{2}+\frac{3 x^{3}}{2}-\frac{x^{4}}{2}
$$

is

$$
u(x)=x^{2}-x^{3}
$$

By the same manipulations as in the previous example and assuming $m=3$, we have

$$
c_{0}=\frac{1}{12}, \quad c_{1}=\frac{1}{20}, \quad c_{3}=-\frac{1}{12}, \quad c_{4}=-\frac{1}{20} .
$$

Thus,

$$
\begin{aligned}
u(x) & =\left(\begin{array}{llll}
\frac{1}{12} & \frac{1}{20} & -\frac{1}{12} & -\frac{1}{20}
\end{array}\right)\left(\begin{array}{c}
1 \\
2 x-1 \\
6 x^{2}-6 x+1 \\
20 x^{3}-30 x^{2}+12 x-1
\end{array}\right) \\
& =x^{2}-x^{3}
\end{aligned}
$$

which is the exact solution (35).

Example 3. We next consider the linear singular two-point BVPs [11],

$$
u^{\prime \prime}(x)+\frac{1}{x} u^{\prime}(x)=g(x), \quad 0<x \leq 1,
$$

subject to the boundary conditions

$$
u^{\prime}(0)=0, \quad u(1)=\cos 1
$$

The exact solution of (38) subject to (39) in the case

$$
g(x)=-\cos x-\frac{1}{x} \sin x
$$

is

$$
u(x)=\cos x
$$

By using the technique described in Section 3.1, with $m=$ 9 and $m=8$, the values of the unknown matrix $C^{T}$ are given in Table 1. Figure 1 shows the absolute errors of $u(x)$ in the interval $x \in[0,1]$ for different values of $m$. Obviously, increasing the number of terms of the Legendre polynomials has the effect of increasing the solution accuracy.

\section{Numerical Experiments}

Example 4. Finally, consider the nonlinear singular twopoint BVP [12]

$$
u^{\prime \prime}(x)+\frac{2}{x} u^{\prime}(x)+(u(x))^{5}=g(x), \quad 0<x<1
$$

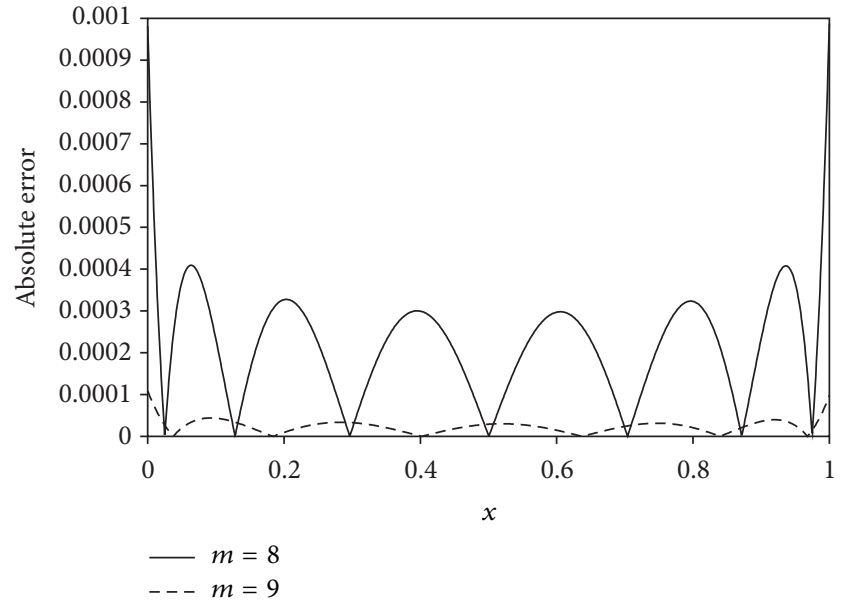

Figure 1: Absolute errors of $u(x)$ in the interval $x \in[0,1]$ for different values of $m$ of Example 3 .

TABLE 1: The values of the unknown matrix $C^{T}$ for $m=8$ and $m=9$ of Example 3.

\begin{tabular}{lll}
\hline$c_{i}$ & $m=9$ & $m=8$ \\
\hline$c_{0}$ & 0.8414709848 & 0.8414709848 \\
$c_{1}$ & -0.2337732110 & -0.233773211 \\
$c_{2}$ & $-0.718349800 E-1$ & $-0.7183498 E-1$ \\
$c_{3}$ & $0.394000000 E-2$ & $0.394000000 E-2$ \\
$c_{4}$ & $0.512000000 E-3$ & $0.512000000 E-3$ \\
$c_{5}$ & 0.0000000000 & 0.0000000000 \\
$c_{6}$ & 0.0000000000 & 0.0000000000 \\
$c_{7}$ & $-0.100000000 E-2$ & $0.100000000 E-3$ \\
$c_{8}$ & 0.0000000000 & 0.0000000000 \\
$c_{9}$ & 0.0000000000 & \\
\hline
\end{tabular}

subject to the boundary conditions

$$
u(0)=1, \quad u(1)=\frac{\sqrt{3}}{2} .
$$

The exact solution of (42) subject to (43) in the case $g(x)=0$ is

$$
u(x)=\frac{1}{\sqrt{1+\left(x^{2} / 3\right)}} .
$$

We approximate the solution as

$$
u(x)=\sum_{j=0}^{10} c_{j} P_{j}(x)=C^{T} \phi(x) .
$$

Here, $D^{1}$ and $\left(D^{1}\right)^{2}$ are as given in (27). Using (19), we have

$$
C^{T}\left(D^{1}\right)^{2} \phi(x)+\frac{2}{x} C^{T} D^{1} \phi(x)+\left(C^{T} \phi(x)\right)^{5}=0 .
$$

Now, we collocate $(46)$ at the first nine roots of $P_{11}(x)$, that is

$$
\begin{gathered}
x_{0} \approx 0.01088567093, \quad x_{1} \approx 0.05646870012, \ldots, \\
x_{8} \approx 0.8650760028 .
\end{gathered}
$$


TABLE 2: The values of the unknown matrix $C^{T}$ for $m=8, m=9$, and $m=10$ of Example 4 .

\begin{tabular}{lccc}
\hline$c_{i}$ & $m=10$ & $m=9$ & $m=8$ \\
\hline$c_{0}$ & $9.514261509 E-01$ & $9.514261511 E-01$ & $9.514261551 E-01$ \\
$c_{1}$ & $-6.966876186 E-02$ & $-6.966876151 E-02$ & $-6.966879907 E-02$ \\
$c_{2}$ & $-1.857026292 E-02$ & $-1.857026217 E-02$ & $-1.857018754 E-02$ \\
$c_{3}$ & $2.743692722 E-03$ & $2.743693272 E-03$ & $2.743858911 E-03$ \\
$c_{4}$ & $1.552129543 E-04$ & $1.552138998 E-04$ & $1.549045730 E-04$ \\
$c_{5}$ & $-6.327914767 E-05$ & $-6.327882993 E-05$ & $-6.349471339 E-05$ \\
$c_{6}$ & $1.707748370 E-06$ & $1.708463078 E-06$ & $1.940268030 E-06$ \\
$c_{7}$ & $1.062085947 E-06$ & $1.061 F 824274 E-06$ & $1.136868997 E-06$ \\
$c_{8}$ & $-1.096567492 E-07$ & $-1.092619480 E-07$ & $-1.103651304 E-07$ \\
$c_{9}$ & $-1.180570173 E-08$ & $-1.275316483 E-08$ & \\
$c_{10}$ & $2.978752464 E-09$ & & \\
\hline
\end{tabular}

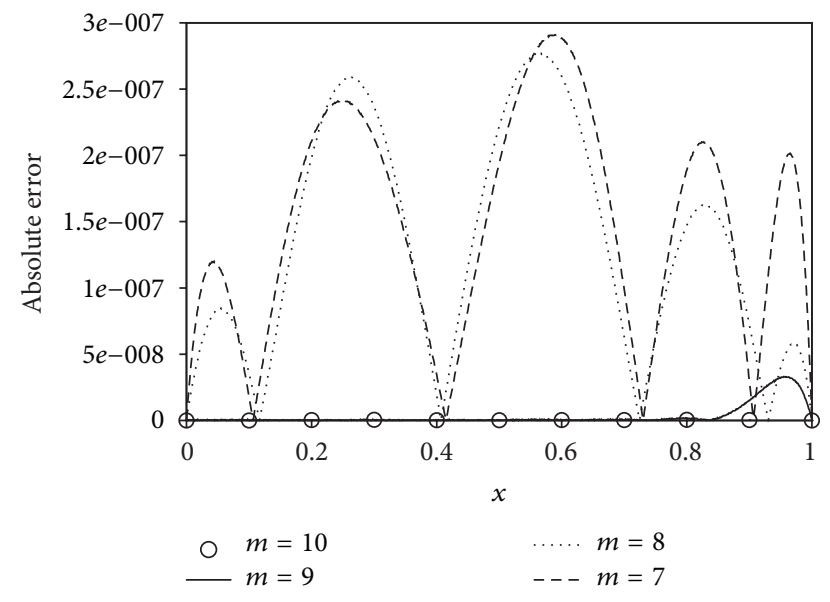

FIGURE 2: Absolute errors of $u(x)$ in the interval $x \in[0,1]$ for different values of $m$ of Example 4 .

Also (21) gives

$$
\begin{aligned}
C^{T} \phi(0)= & c_{0}-c_{1}+c_{2}-c_{3}+c_{4}-c_{5} \\
& +c_{6}-c_{7}+c_{8}-c_{9}+c_{10}=1, \\
C^{T} \phi(1)= & c_{0}+c_{1}+c_{2}+c_{3}+c_{4}+c_{5} \\
& +c_{6}+c_{7}+c_{8}+c_{9}+c_{10}=\frac{\sqrt{3}}{2} .
\end{aligned}
$$

Equations (46) and (48) generate 11 nonlinear equations which can be solved using Newton's iterative method. The values of the unknown matrix $C^{T}$ for $m=8, m=9$, and $m=10$ are given in Table 2 . Figure 2 shows the absolute errors of $u(x)$ in the interval $x \in[0,1]$ for different values of $m$.

\section{Conclusions}

In this paper, the Legendre operational matrix of derivative was applied to solve a class of linear and nonlinear singular two-point BVPs. Different from other numerical techniques, only a small size operational matrix is required to provide the solution at high accuracy. It can be clearly seen in the paper that the proposed method is working well even with a few number of terms of the Legendre polynomials.

\section{Acknowledgment}

This work was supported by the Universiti Kebangsaan Malaysia's Grant no. DIP-2012-12.

\section{References}

[1] A. S. Bataineh, M. S. M. Noorani, and I. Hashim, "Approximate solutions of singular two-point BVPs by modified homotopy analysis method," Physics Letters A, vol. 372, no. 22, pp. 40624066, 2008.

[2] A. S. V. Ravi Kanth and K. Aruna, "Solution of singular twopoint boundary value problems using differential transformation method," Physics Letters A, vol. 372, no. 26, pp. 4671-4673, 2008.

[3] J. Lu, "Variational iteration method for solving two-point boundary value problems," Journal of Computational and Applied Mathematics, vol. 207, no. 1, pp. 92-95, 2007.

[4] I. T. Abu-Zaid and M. A. Gebeily, "A finite difference method for approximating the solution of a certain class of singular twopoint boundary value problems," Arabian Journal of Mathematical Science, vol. 1, pp. 25-29, 1995.

[5] A. S. V. Ravi Kanth and Y. N. Reddy, "Cubic spline for a class of singular two-point boundary value problems," Applied Mathematics and Computation, vol. 170, no. 2, pp. 733-740, 2005.

[6] A. Saadatmandi and M. Dehghan, "A new operational matrix for solving fractional-order differential equations," Computers \& Mathematics with Applications, vol. 59, no. 3, pp. 1326-1336, 2010.

[7] R. K. Pandey, N. Kumar, A. Bhardwaj, and G. Dutta, "Solution of Lane-Emden type equations using Legendre operational matrix of differentiation," Applied Mathematics and Computation, vol. 218, no. 14, pp. 7629-7637, 2012.

[8] S. Kazem, S. Abbasbandy, and S. Kumar, "Fractional-order Legendre functions for solving fractional-order differential equations," Applied Mathematical Modelling, vol. 37, no. 7, pp. 5498-5510, 2013. 
[9] C. Canuto, M. Y. Hussaini, A. Quarteroni, and T. A. Zang, Spectral Methods in Fluid Dynamics, Prentice Hall, New Jersey, NJ, USA, 1988.

[10] M. Cui and F. Geng, "Solving singular two-point boundary value problem in reproducing kernel space," Journal of Computational and Applied Mathematics, vol. 205, no. 1, pp. 6-15, 2007.

[11] M. Kumar, "A difference scheme based on non-uniform mesh for singular two-point boundary value problems," Applied Mathematics and Computation, vol. 136, no. 2-3, pp. 281-288, 2003.

[12] R. Qu and R. Agarwal, "A collocation method for solving a class of singular nonlinear two-point boundary value problems," Journal of Computational and Applied Mathematics, vol. 83, no. 2, pp. 147-163, 1997. 


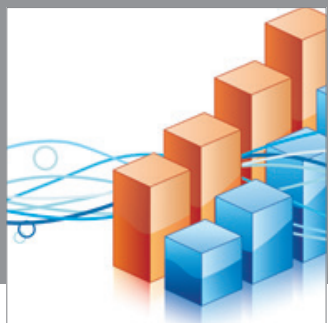

Advances in

Operations Research

mansans

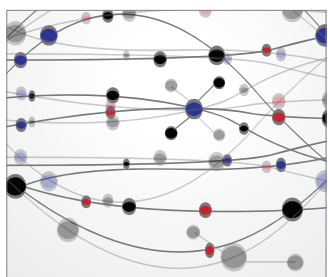

The Scientific World Journal
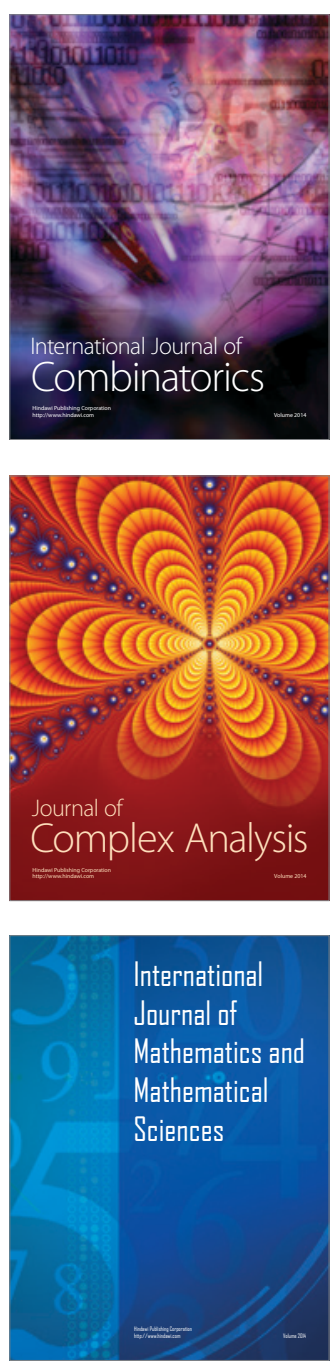
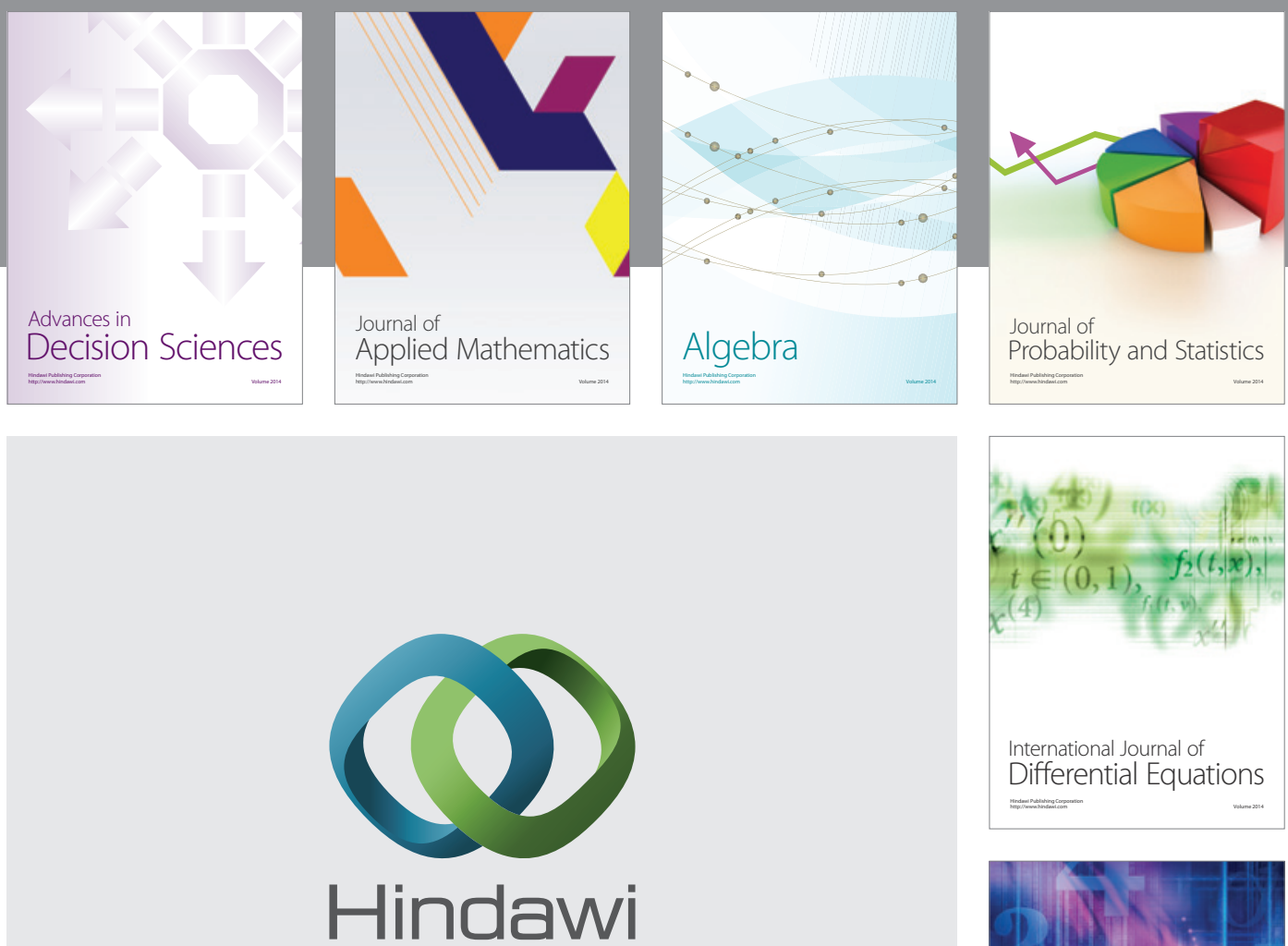

Submit your manuscripts at http://www.hindawi.com
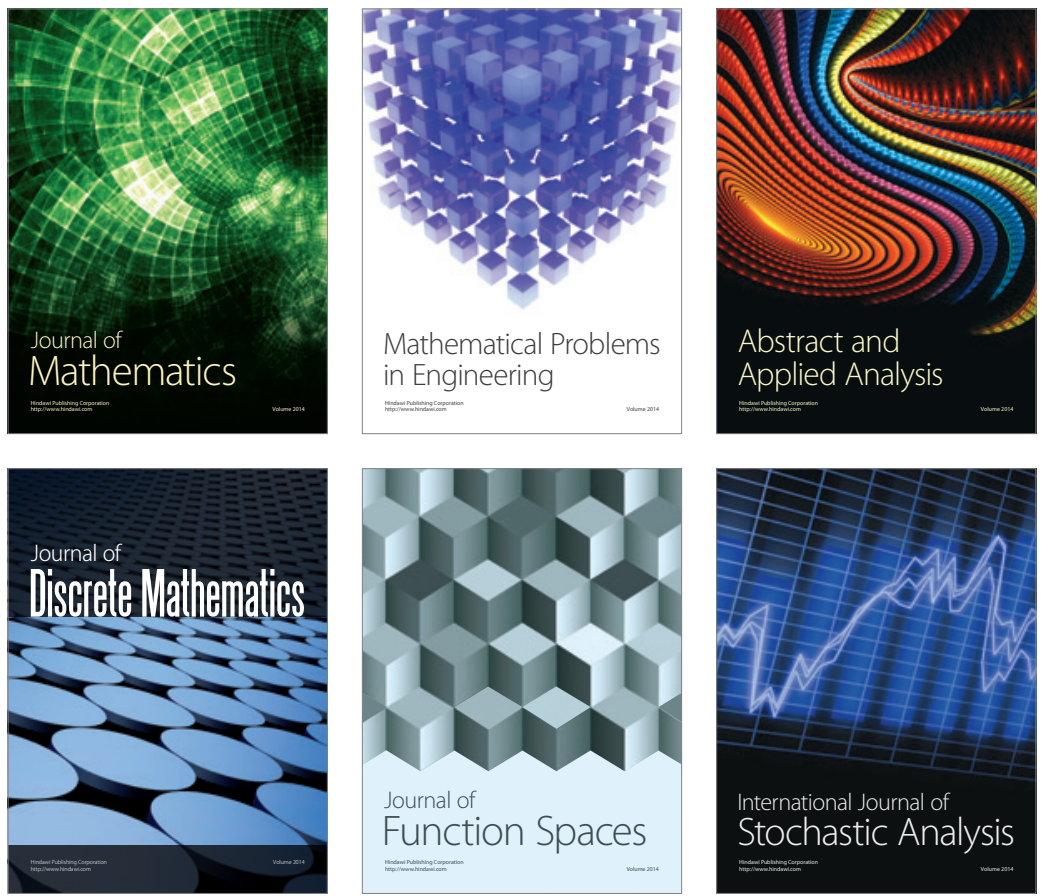

Journal of

Function Spaces

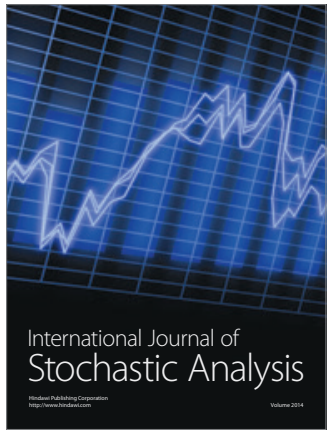

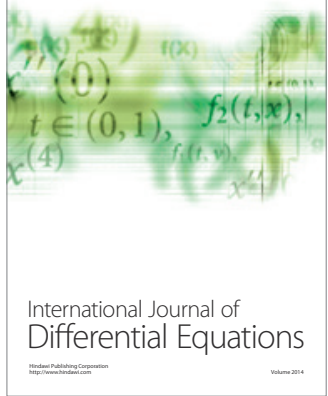
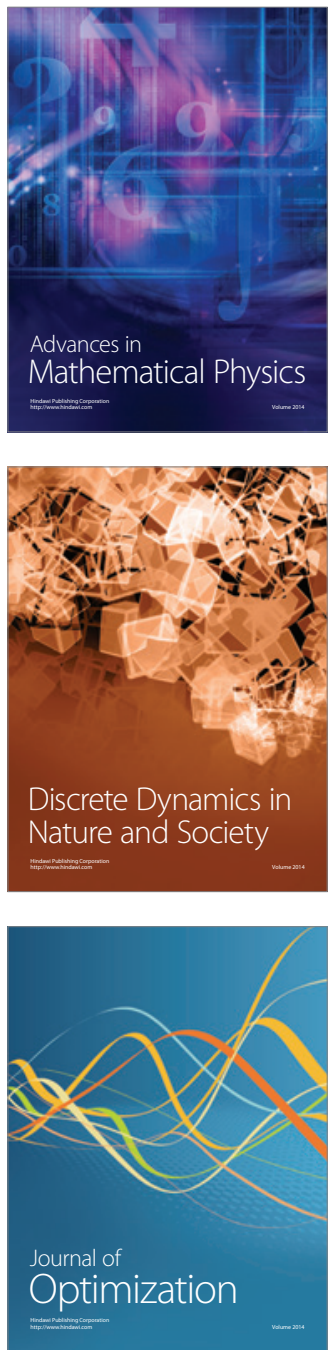